\title{
Prediction of Protein Functions Using Protein Interaction Data
}

\author{
Haemoon Jung and Kyungsook Han* \\ School of Computer Science and Engineering, Inha University, Inchon 402-751, Korea \\ idjhmoon@hotmail.com, khan@inha.ac.kr
}

\begin{abstract}
Information on protein-protein interactions provides valuable insight into the underlying mechanism of protein functions. However, the accuracy of protein-protein interaction data obtained by high-throughput experimental methods is low, and thus requires a rigorous assessment of their reliability. This paper proposes a computational method for predicting the unknown function of a protein interacting with a protein with known function, and presents the experimental results of applying the method to the protein-protein interactions in yeast and human. This method can also be used to assess the reliability of the protein-protein interaction data.
\end{abstract}

\section{Introduction}

High-throughput experimental techniques enable the study of protein-protein interactions at the proteome scale through systematic identification of physical interactions among all proteins in an organism [1]. The increasing volume of proteinprotein interaction data is becoming the foundation for new biological discoveries. Protein-protein interactions play important roles in nearly all events that take place in a cell [1]. Particularly valuable will be analyses of proteins that play pivotal roles in biological phenomena in which the physiological interactions of many proteins are involved in the construction of biological pathways, such as metabolic and signal transduction pathways [2].

Here is a more elaborate example. All biochemical process is regulated through protein function. Therefore, diseases (hereditary/non-hereditary) are made manifest as a result of protein function. Also, finding protein function is necessary for the normal progression of medicinal development. Thus, with the completion of genome sequencing of several organisms, the functional annotation of the proteins is of most importance [3]. This function is considered as property of sequence or structure.

Several research groups have developed methods for functional annotation. The classical way is to find homologies between a protein and other proteins in databases using programs such as FASTA and PSI-BLAST, and then predict functions [3]. Another approach is called the Rosetta stone method, where two proteins are inferred to interact if they are together in another genome [3]. We propose a method for determining the reliability of the protein-protein interaction data obtained by high-

* To whom correspondence should be addressed. Email: khan@inha.ac.kr

M. Bubak et al. (Eds.): ICCS 2004, LNCS 3037, pp. 317-324, 2004.

(C) Springer-Verlag Berlin Heidelberg 2004 
throughput experimental methods. This method can also be used for predicting the unknown function of a protein interacting with a protein with known function.

\section{Analysis of Yeast Protein Data}

\subsection{Data Sets}

The data of yeast protein interactions were obtained from the Comprehensive Yeast Genome Database (CYGD) at MIPS [7]. After removing 1,150 redundant data, 9,490 non-redundant interactions were left. In addition to the interaction data, the information on the yeast protein catalogue such as class, enzyme code number, motif, function, complex and cell localization were extracted. Table 1 shows the number of yeast protein entries in each catalogue.

Table 1. Yeast protein catalogues

\begin{tabular}{lc}
\hline Protein catalogues & Number of protein entries \\
\hline Classes & 1,070 \\
Complexes & 1,321 \\
EC number & 1,152 \\
Motifs & 2,539 \\
Phenotype & 3,120 \\
Function & 11,506 \\
Cell localization & 2,407 \\
\hline
\end{tabular}

The function catalogue in particular is important because the primary focus of this study is the prediction of protein functions. We shall now examine the function catalogue more closely.

Functions of yeast proteins were all arranged from FunCat (The Functional Catalogue) of MIPS. The FunCat is an annotation scheme for the functional description of proteins from prokaryotes, unicellular eukaryotes, plants and animals [7]. Taking into account the broad and highly diverse spectrum of known protein functions, the FunCat consists of 30 main functional categories (or branches) that cover general fields like cellular transport, metabolism and signal transduction [7]. The main branches exhibit a hierarchical, tree like structure with up to six levels of increasing specificity [7]. In total, the FunCat has 1,445 functional categories. Among of them, 215 functional categories were applied to yeast. Tables 2 and 3 show the number of yeast protein entries and yeast proteins, respectively, in each functional category of FunCat. 
Table 2. The number of yeast proteins in each function category

\begin{tabular}{lc}
\hline \multicolumn{1}{c}{ Function category } & Number of yeast proteins \\
\hline Unclassified function & 2,399 \\
uncleus & 764 \\
cytoplasm & 554 \\
mitochondrion & 366 \\
transcriptional control & 334 \\
mitotic cell cycle and cell cycle control & 326 \\
C-compound and & 260 \\
carbohydrate utilization & 215 \\
ribosome biogenesis & 175 \\
stress response & 170 \\
budding, & 167 \\
cell polarity and filament formation & 160 \\
protein modification & 157 \\
pheromone response, mating-type & 146 \\
determination, sex-specific proteins & 145 \\
endoplasmic reticulum & 129 \\
protein targeting, & 120 \\
sorting and translocation & \\
plasma membrane & 15 \\
vesicular transport (Golgi network, etc.) & 1 \\
regulation of C-compound and & \\
carbohydrate utilization & $\ldots$
\end{tabular}

\subsection{Analysis Results}

The protein interaction data in each catalogue of Table 1 were analyzed. As shown in Fig. 1, the proteins in a same complex interact with the highest probability $(0.33)$, and the proteins with a same function interact with the second highest probability $(0.28)$. The proteins in a same complex interact and with a same function interact with the probability of 0.39 , which is higher than 0.33 and 0.28 but not much higher. This implies that a large portion of the proteins in the two catalogues overlap.

The ratio of the number of interactions between proteins in a same complex to the total number of interactions is 0.25 . The ratio of the number interactions between proteins with a same function to the number of interactions between proteins in a same complex is 0.97 . This indicates that interacting proteins in a same complex also have a same function with a high probability. The inverse is not necessarily true. Consequently, we discovered an association rule: If proteins $\mathrm{A}$ and $\mathrm{B}$ are in a same complex and interact each other, proteins $\mathrm{A}$ and $\mathrm{B}$ have a same function. 
Table 3. Scheme of yeast function catalogue

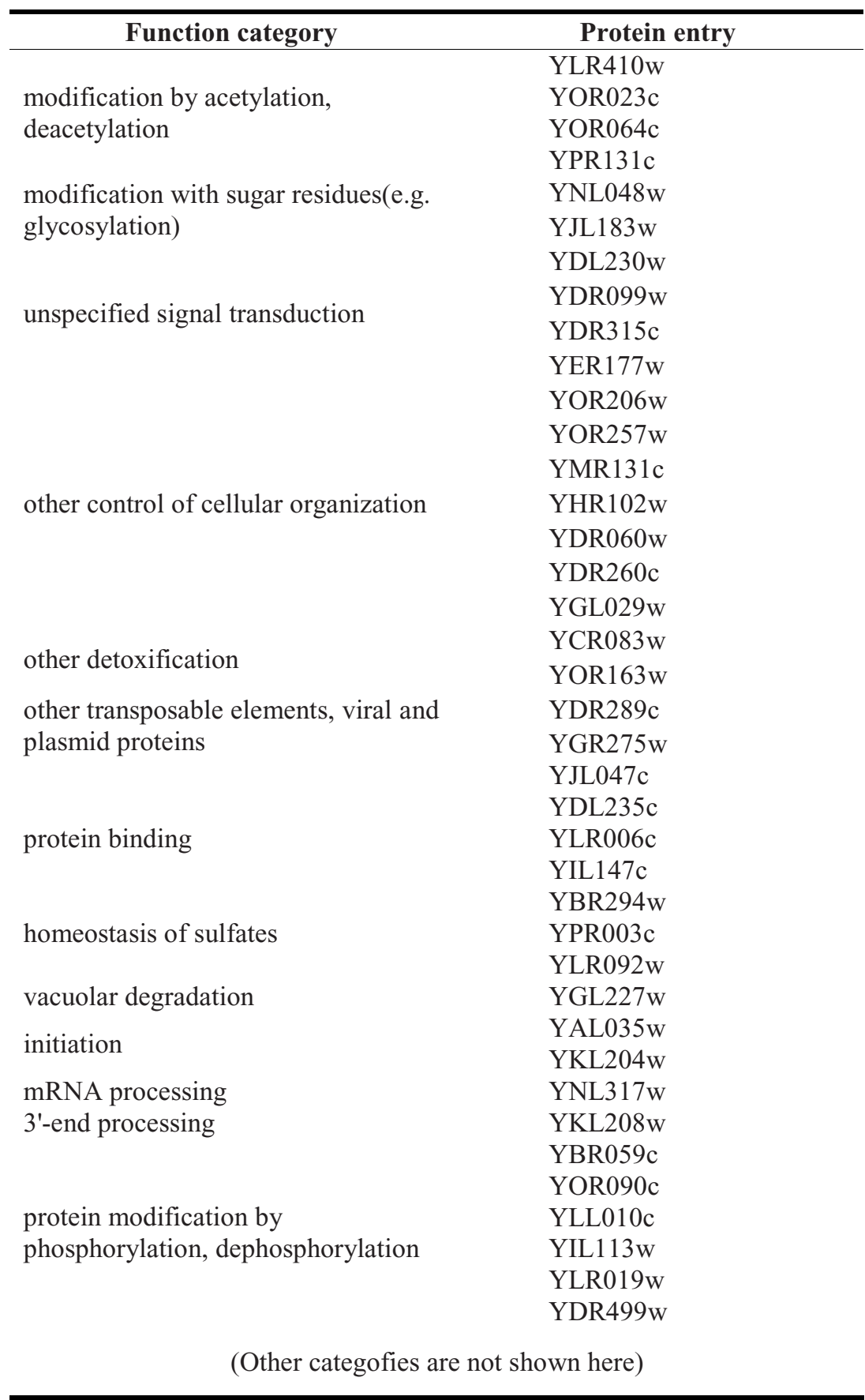




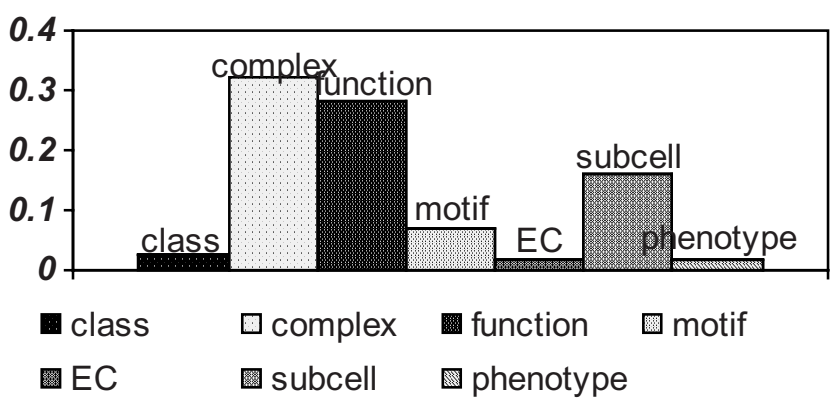

Fig. 1. The probability of interaction between proteins in each catalogue.

This rule is also confirmed in the protein-protein interaction networks of Fig. 2. Fig. 2A shows a network of interactions between proteins in a same complex, visualized by InterViewer [4, 5]. Interacting proteins with a same function are selected from this network and shown in green. Most nodes of Fig. 2A are selected as proteins involved with the interactions between proteins with a same function. Figure 2B shows a network of interactions between proteins with a same function. Interacting proteins in a same complex are selected from this network and shown in green, too. Only a small portion of the entire nodes is selected.

\section{Prediction of Human Protein Function}

Uetz et al. [8] and Ito et al. [9] show that the function of uncharacterized proteins can be predicted in the light of the interacting partner by using the principle of 'guilt by association', which maintains that two interacting proteins are likely to participate in the same cellular function $[2,6]$.

Since the function of many human proteins is unknown, we predicted the function of human proteins. Suppose that $\mathrm{SP}_{\mathrm{j}}$ is a protein with unknown function, $\mathrm{P}_{\mathrm{i}}$ is a protein interacting with $\mathrm{SP}_{\mathrm{j}}$, AF is the total number of functions of interacting proteins, $\mathrm{AP}$ is the total number of interacting proteins, and that $\mathrm{DP}_{\mathrm{i}}$ is the degree of a particular partner protein. Then the score function for assessing the probability that protein $\mathrm{SP}_{\mathrm{j}}$ has the same function with protein $\mathrm{P}_{\mathrm{i}}$ can be computed by equation 1 .

$$
\operatorname{Score}(j, i)=D P_{i} \times \frac{1}{A F} \times \frac{1}{A P} \times C P_{i}
$$

where $\mathrm{CP}_{\mathrm{i}}$ is a complex constant. Algorithms 1-3 describe how to predict the function of a protein. In the algorithms, protein_num is the number of gathered proteins, and cnt represents the number of interacting partners with a same function. All interaction data are cleaned first by Algorithm 2, and the function of interacting partners is counted in Algorithm 3. 

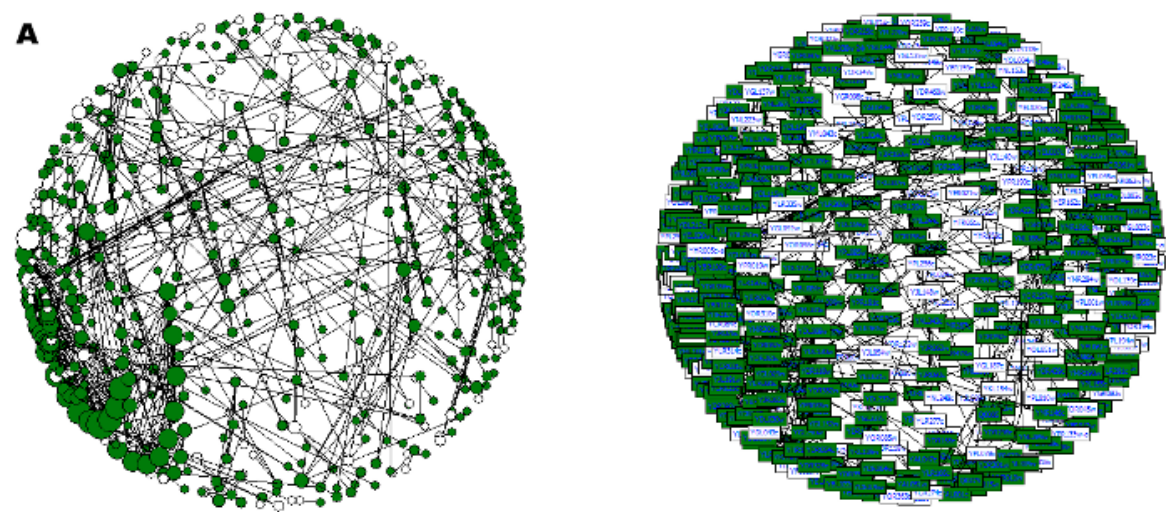

$\mathbf{B}$
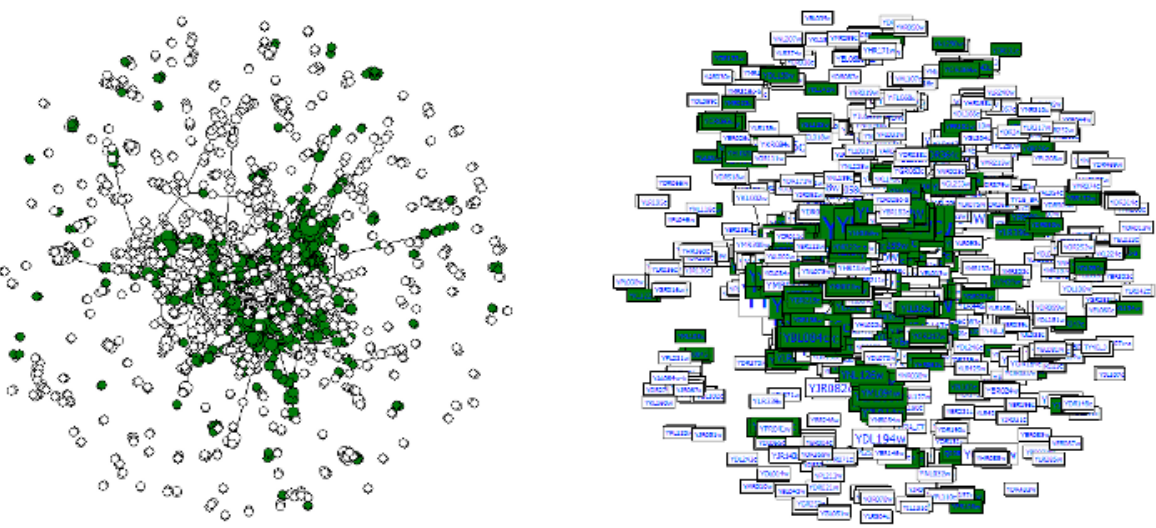

Fig. 2. (A) Left: A network of interactions between proteins in a same complex. Interacting proteins with a same function are shown in green. The network in the right shows the same network in the left but with node labels shown. (B) Left: A network of interactions between proteins with a same function. Interacting proteins in a same complex are shown in green. The network in the right shows the same network in the left but with node labels shown.

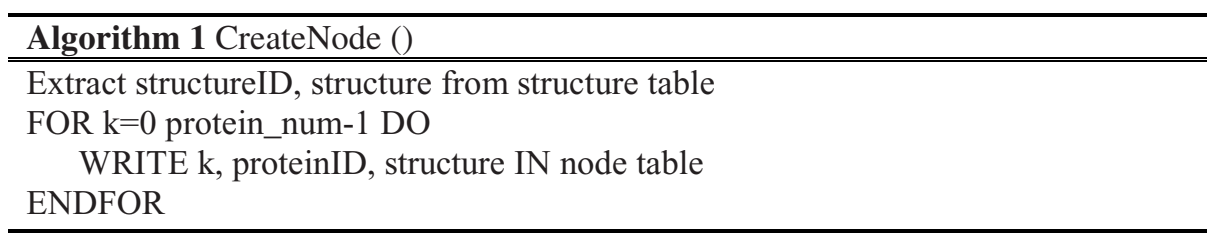

\begin{tabular}{l}
\hline Algorithm 2 ClearInteract $($ ) \\
\hline \hline Extract source, target from interactions \\
FOR $\mathrm{k}=0$ protein_num-1 DO \\
IF (source $==\mathrm{k}$ ) THEN \\
WRITE source, target IN non-redundant_interaction \\
ELSEIF (target $==\mathrm{k}$ ) THEN \\
\hline
\end{tabular}




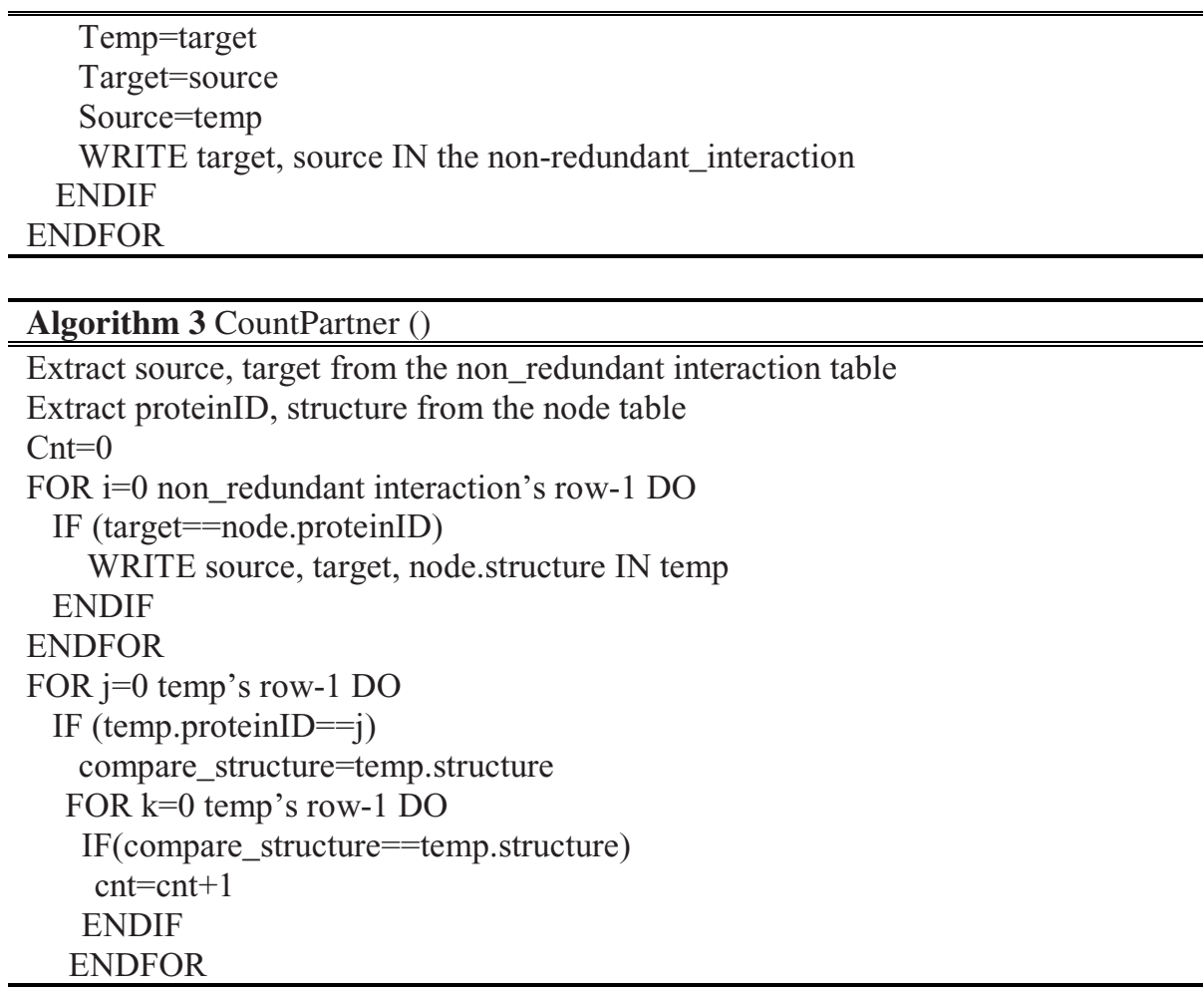

Table 4 summarizes the prediction results. The probability of interaction between proteins in each category is different from that in yeast proteins.

Table 4. The probability of interaction between human proteins

\begin{tabular}{lc}
\hline Items & Value \\
\hline $\begin{array}{l}\text { Ratio of the interactions between proteins in a same complex } \\
\text { to the total interactions }\end{array}$ & 0.26 \\
Ration of the interactions between proteins with a same & 0.34 \\
function & \\
to the total interactions & 0.3547 \\
Ratio of interactions between proteins with a same function \\
to the interactions between proteins in a same complex \\
$\begin{array}{l}\text { Ratio of interactions between proteins in a same complex } \\
\text { to the interactions between proteins with a same function }\end{array}$
\end{tabular}

\section{Conclusion}

Proteins have many different biological functions by interacting with other proteins, and two interacting proteins are likely to have same biological function. Therefore, if 
a protein with known function is identified to interact with an uncharacterized protein, the function of the uncharacterized protein can be predicted. From the analysis of the experimental data of yeast protein interactions, we discovered a reliable association rule that "if proteins A and B exist in a same complex and interact each other, proteins A and B have a same function." We have developed an algorithm for predicting the function of proteins based on the association rule, and applied the algorithm to the human protein interaction data. Experimental results show that the algorithm is potentially useful for predicting the function of uncharacterized proteins.

Acknowledgements. This work was supported by the Ministry of Information and Communication of Korea under grant IMT2000-C3-4.

\section{References}

1. Chen, Y., Xu, D.: Computational analyses of high-throughput protein-protein interaction data. Current protein and peptide science 4 (2003) 159-180

2. Saito, R., Suzuki, H., Hayashizaki, Y.: Interaction generality, a measurement to assess the reliability of a protein-protein interaction. Nucleic Acids Research 30 (2002) 1163-1168

3. Deng, M., Zhang, K., Mehta, S., Chen, T., Sun, F.: Prediction of Protein Function Using Protein-Protein Interaction Data. IEEE Computer Society Bioinformatics Conference (2002) 197-206

4. Ju, B., Park, B., Park, J., Han, K.: Visualization and analysis of protein interactions. Bioinformatics 19 (2003) 317-318

5. Han, K., Ju, B.: A fast layout algorithm for protein interaction networks. Bioinformatics 19 (2003) 1882-1888

6. Oliver, S.: Guilt-by-association goes global. Nature 403 (2000) 601-603.

7. CYGD Home Page http://mips.gsf.de/genre/proj/yeast/index.jsp

8. Uetz, P., Giot, L., Cagney, G., Mansfield, T.A., Judson, R.S., Knight, J.R., Lockshon, D., Narayan, V., Srinivasan, M., Pochart, P. et al.: A comprehensive analysis of protein-protein interactions in Saccharomyces cerevisiae. Nature (2000) 403 623-627.

9. Ito, T., Chiba, T., Ozawa, R., Yoshida, M., Hattori, M., Sakaki, Y.: A comprehensive twohybrid analysis to explore the yeast protein interactome. Proc Natl Acad Sci USA (2001) 98 4569-4574 